\title{
Editor's Message to Special Issue on Internet and Operation Technology from Risk Management Perspective
}

\author{
Kensuke Miyashita $^{1, a)}$
}

This special issue is planned and edited by the Special Interest Group on Internet and Operation Technology (SIG-IOT) as a central role.

On 11 March 2011, the earthquake off the Pacific coast of Tōhoku occurred. At the time of proposal for this special issue, reconstruction after the earthquake and tsunami had continued through nine months after the occurrence. For network researchers, network engineers and system administrators, it is worth considering what they have done directly after the earthquake and what they should do. It also leads us to think what trouble of network or computer system we should suppose in ordinary operations. In this special issue, we have made a concerted effort to attract excellent papers discussing problems concerned in operation technology of network and computer system from risk management perspective with a lot of experiences and practices after the earthquake as a beginning point of discussion.

We, 24 members of editorial board, received 25 papers and reviewed them thoroughly and rigorously. This special issue has common themes in the 4th Internet and Operation Technology Symposium (IOTS2011) held in December 2011, so we include members of the program committee of IOTS2011 to reinforce the continuity of theme. In the same way of former special issues arranged by SIG-IOT, we have made a best effort to give precedence to "Dos and Don'ts" (established by the editorial committee of IPSJ Journal) and asked the reviewers to make comments instructive for authors. Consequently, we selected 13 papers to be included in the special issue. The presented papers are mainly devoted to discussion on direct measures against the disaster and planned outage, inventive ideas from ordinary operations of network and computer system, and improvements of network security.

In this special issue, we invited a paper written by Mr. Naoto Matsumoto (SAKURA Internet Inc.) who is a leading person to support the spread of information about situation of damage and infrastructures on WWW after the earthquake. In this paper, he mainly analyses the disclosure of information from his experience and advocates reducing impact of information system damaged from disasters.

The author wishes to express his thanks to whom it may concern in IPSJ, the authors who submit excellent papers, and especially Mr. Naoto Matsumoto for the invited paper. The author also

Faculty for the Study of Contemporary Society, Kyoto Women's University, Kyoto 605-8501, Japan

a) miyasita@cs.kyoto-wu.ac.jp thanks the reviewers for rigorous reviews and instructive comments, and thanks the editorial board members of this special issue for the continued encouragement, guidance and support in the preparation of this issue.

Hopefully, this special issue will contribute to make the Internet truly dependable infrastructure.

\section{The Editorial Committee}

- Editor in-Chief:

Editor in-Chief: Kensuke Miyashita (Kyoto Women's Univ.)

- Deputy Chief Editor:

Deputy Chief Editor: Akira Sato (Tsukuba Univ.)

\section{- Editorial Committee:}

Toyokazu Akiyama (Kyoto Sangyo Univ.)

Koji Ando (mokha Inc.)

Shunsuke Fujieda (Tokyo Univ. of Tokyo)

Yoichi Hagiwara (Tokyo Univ. of Agriculture and Technology)

Haruhisa Hayashi (Univ. of Hyogo)

Yutaka Hisanaga (Yamaguchi Univ.)

Hayato Ishibashi (Osaka City Univ.)

Dai Ishijima (Technology Research Institute of Osaka Prefecture)

Eiji Kawai (National Institute of Information and Communications Technology)

Ryozo Kiyohara (Kanagawa Institute of Technology)

Hideo Masuda (Kyoto Institute of Technology)

Motonori Nakamura (National Institute of Infomatics)

Kouji Nishimura (Hiroshima Univ.)

Nobuo Ogashiwa (Maebashi Kyoai Gakuen College)

Makoto Otani (Saga Univ.)

Akinori Saito (Tottori Univ. of Environmental Studies)

Shu Sakashita (Acutus Software, Inc.)

Mikifumi Shikida (Japan Advanced Institute of Science and Technology)

Kazuya Suzuki (NEC Corporation)

Nariyoshi Yamai (Okayama Univ.)

Takashi Yamanoue (Kagoshima Univ.)

Kazuyuki Yoshida (Oita Univ.) 